\title{
Relationship between the Quality of Sleep and Psychiatric Morbidity among the Patients of Head and Neck Tumours
}

\author{
Usama Bin Zubair1, Humza Mumtaz² and Rizwan Taj ${ }^{1}$
}

\begin{abstract}
Objective: To assess the difference between mean sleep (PSQI) scores in the patients with head and neck tumours with and without the psychiatric morbidity; and assess the associated socio-demographic factors.

Study Design: Descriptive cross-sectional study.

Place and Duration of Study: Pakistan Institute of Medical Sciences (PIMS), Islamabad, from May to November 2017.

Methodology: One hundred and seventy patients suffering from the tumours of head and neck region were analysed in this study, which were treated in the E.N.T Department. Pittsburgh Sleep Quality Index (PSQI) was used to record the sleep quality of the study participants. General Health Questionnaire 12 (GHQ-12) was used to look for the presence of psychiatric morbidity among these patients. Mean sleep scores were compared in the patients with and without the presence of psychiatric morbidity. Relationship of the socio-demographic factors was also studied with the presence of poor sleep quality among the study participants.

Results: A total of 170 patients filled the PSQI; out of them, $58(34.1 \%)$ had scores lying within the range of normal sleep quality while $112(65.9 \%)$ had insufficient quality of sleep. One hundred and eight (63.5\%) patients had significant psychiatric morbidity; whereas, $62(36.5 \%)$ had no psychiatric morbidity on GHQ-12. Mean PSQI score in patients without psychiatric morbidity was $(6.2 \pm 3.2)$ while in the patients with psychiatric morbidity was $9.4 \pm 4.6(p<0.001)$.

Conclusion: Patients suffering from head and neck tumours showed high frequency of both poor sleep quality and psychiatric morbidity. Patients with presence of psychiatric morbidity or who had undergone surgical resection of tumour should be routinely screened for the sleep problems.
\end{abstract}

Key Words: Sleep quality, Head and neck tumours, Psychiatric morbidity.

\section{INTRODUCTION}

Around $10-15 \%$ of the general population face sleep problems of one kind or another, making them a common healthcare problem in all parts of the world. ${ }^{1}$ Various types of sleep problems include bad quality of sleep, sleep apnea and insomnia, which can be part of various physical problems including pregnancy, ${ }^{2}$ chronic renal failure ${ }^{3}$ malignancies, 4 and various mental health problems, 5 etc.

Head and neck tumours are aggressive tumours that account for approximately $3 \%$ of all the diagnosed cases of malignancies. 6 Whole physiology of human body is disturbed in one way or another when a patient is having a deadly disease like cancer. Sleep, appetite and sexual function are altered invariably in patients suffering from cancer. ${ }^{7}$

Disturbed sleep quality among the patients of head and neck tumours have been reported globally. in a study

1 Department of Psychiatry, Pakistan Institute of Medical Sciences (PIMS), SZABMU, Islamabad, Pakistan

2 Department of ENT, Combined Military Hospital, Rawalpindi, Pakistan

Correspondence: Dr. Usama Bin Zubair, Department of Psychiatry, Pakistan Institute of Medical Sciences (PIMS), SZABMU, Islamabad, Pakistan

E-mail: drusamabinzubair@yahoo.com

Received: July 18, 2018; Accepted: January 18, 2019 done in Netherlands, $56 \%$ of the patients of head and neck tumours had disturbed sleep. 8 Another similar study showed that insomnia was among the top four complains reported by the patients of head and neck tumours. ${ }^{9}$ Literature reported by Faiz et al. revealed that daytime fatigue, sleepiness and sleep-related breathing disorders were common in patients with tumours in the head and neck region. ${ }^{10}$ Sleep related problems, if persist for long, have a direct effect on mental health and overall quality of life of a patient who is already suffering from a devastating illness. ${ }^{9,11}$

Many studies done in the past have shown a strong relationship between sleep and other mental health problems. Shuman et al. concluded that depression was present among $49 \%$ of the patients of head and neck tumours and patients with depression significantly reported more sleep problems as compared to patients who were not depressed.12 Another recent study revealed that there was a significant and powerful correlation between mental health with sleep quality of pulmonology patients. ${ }^{13} \mathrm{~A}$ study done in USA among the patients of advanced cancer concluded that increasing depression was positively correlated to worse subjective sleep quality. ${ }^{14}$

Physical effects of head and neck tumours may contribute to the poor sleep quality among the patients but chronic nature of disease and other disease-related factors may prone the individuals towards various mental health disorders, which can affect the sleep quality and overall 
quality of life. Quality of sleep and mental health problems have been studied in the past separately among the patients of head and neck tumours; but no local study is available to establish the relationship between these two parameters.

The objective of this research was to find out the relationship between quality of sleep and psychiatric well-being among the sufferers of malignancies of head and neck region.

\section{METHODOLOGY}

This descriptive study was carried out at the Departments of E.N.T and Psychiatry at Pakistan Institute of Medical Sciences (PIMS), Islamabad, from May to November 2017. WHO sample size calculator was used to calculate the required sample size for this study. ${ }^{12}$ Nonprobability consecutive sampling technique was applied to gather the sample. Patients from 18 to 65 years suffering from the head and neck cancer, were included, whether admitted in wards or attending OPD. Other chronic physical illnesses (e.g. diabetes, ischemic heart disease, hypertension, chronic arthritides etc.) or psychiatric illness, either past or current, was the main exclusion criteria. Patients using any kind of illicit substance at present or in past were also excluded. Patients with thyroid or cutaneous cancers or with complain of persistent pain and VAS-core of pain $>6$ or those who were pregnant or unable to read and perform the required questionnaire were also excluded from the study.
After the application of inclusion and exclusion criteria, all the patients suffering from head and neck cancers admitted in ENT ward or presenting in head and neck surgery OPD, were made part of this analysis. Ethical approval was taken from the IRB committee. Written informed consent was taken from the sample population. Detailed history, taking and examination, was performed on all the patients included in this study. CT-scan was used to stage the malignancy.

Pittsburgh Sleep Quality Index (PSQI) was used to assess the quality of sleep. The PSQI is an effective instrument for subjective measure of quality and patterns of sleep. Validated Urdu version of PSQI was applied. Mean PSQI score was compared for the patients with and without the psychiatric morbidity. Psychiatric morbidity and mental health well-being was assessed by using the General Health Questionnaire 12 (GHQ-12). GHQ 12 is a reliable and validated psychometric tool to assess the mental health of an individual. Twelve items are scored from 0 to 3 on Likert scale with cut-off score at three. Personal data of patients were entered in a proforma designed for this study by the combined effort of all. All the questions were asked to assess the condition of participants in last one month.

Statistical Package for Social Sciences version 23.0 was used to analyse the data for this study. Frequencies and percentages for gender and presence of psychiatric morbidity were calculated. Mean \pm SD for age and mean PSQI score of the patients with and without psychiatric morbidity were calculated and independent t-test was

Table I: Characteristics of the study group and their PSQI scores.

\begin{tabular}{|c|c|c|c|c|c|}
\hline \multirow[t]{2}{*}{ Socio-demographic factors } & \multicolumn{2}{|c|}{$\begin{array}{l}\text { Patients with good sleep quality } \\
\text { (PSQI 0-5) }\end{array}$} & \multicolumn{2}{|c|}{$\begin{array}{l}\text { Patients with poor sleep quality } \\
\text { (PSQI >5) }\end{array}$} & \multirow[t]{2}{*}{$\mathrm{p}$-value } \\
\hline & $\mathrm{N}$ & $\%$ & $\mathrm{~N}$ & $\%$ & \\
\hline Total & 58 & 4.1 & 112 & 65.9 & \\
\hline \multicolumn{6}{|l|}{ Family income } \\
\hline Less than outgoings & 31 & 53.4 & 36 & 32.1 & 0.008 \\
\hline More than or equal to outgoings & 27 & 46.6 & 76 & 67.9 & \\
\hline \multicolumn{6}{|l|}{ Psychiatric morbidity } \\
\hline Absent & 33 & 56.9 & 29 & 25.9 & $<0.001$ \\
\hline Present & 25 & 43.1 & 83 & 74.1 & \\
\hline \multicolumn{6}{|c|}{ Presence of feeding or tracheostomy tube } \\
\hline No & 16 & 27.6 & 41 & 36.6 & 0.304 \\
\hline Yes & 42 & 72.4 & 41 & 36.6 & \\
\hline \multicolumn{6}{|l|}{ Tobacco smoking } \\
\hline Non-smoker & 53 & 91.4 & 102 & 91.1 & 1.000 \\
\hline Smoker & 05 & 8.6 & 10 & 8.9 & \\
\hline \multicolumn{6}{|l|}{ Education } \\
\hline Less than $10^{\text {th }}$ grade & 38 & 65.5 & 58 & 51.8 & 0.104 \\
\hline $10^{\text {th }}$ grade or more & 20 & 34.5 & 54 & 48.2 & \\
\hline \multicolumn{6}{|l|}{ On chemotherapy } \\
\hline No & 48 & 82.7 & 89 & 79.5 & 0.686 \\
\hline Yes & 10 & 17.3 & 23 & 20.5 & \\
\hline \multicolumn{6}{|l|}{ On radiotherapy } \\
\hline No & 44 & 75.9 & 87 & 77.7 & 0.848 \\
\hline Yes & 14 & 24.1 & 25 & 22.3 & \\
\hline
\end{tabular}


Table II: The correlated factors relating to quality of sleep: The binary logistic regression.

\begin{tabular}{|c|c|c|c|}
\hline & B & p-value & OR $(95 \% \mathrm{Cl})$ \\
\hline Age (ref. is 55 years or less) & 0.805 & 0.101 & $2.237(0.854-5.862)$ \\
\hline Gender (ref. is male) & 0.625 & 0.270 & $1.868(0.615-5.671)$ \\
\hline Psychiatric morbidity (ref. is absence of psychiatric morbidity) & 2.833 & 0.002 & $17.004(2.797-103.391)$ \\
\hline Marital status (ref. is married) & 1.215 & 0.329 & $0.297(0.026-3.410)$ \\
\hline Family income (ref. is more than or equal to outgoings) & 0.359 & 0.449 & $1.431(0.566-3.622)$ \\
\hline Smoking (ref. is non-smoker) & 0.035 & 0.965 & $1.036(0.217-4.934)$ \\
\hline Education (ref. is above matriculation) & 0.747 & 0.119 & $0.474(0.186-1.211)$ \\
\hline Naswar use (ref. is non-user) & 0.481 & 0.436 & $0.618(0.184-2.072)$ \\
\hline Presence of feeding/tracheostomy tube (ref. is absence of the tubes) & 0.874 & 0.096 & $0.417(0.149-1.168)$ \\
\hline Surgery (ref. is surgery done) & 3.231 & $<0.001$ & $25.30(7.79-82.16)$ \\
\hline Chemotherapy (ref. is no chemotherapy done) & 1.071 & 0.082 & $2.917(0.874-9.732)$ \\
\hline Radiotherapy (ref. is no radiotherapy done) & 0.264 & 0.663 & $0.768(0.234-2.519)$ \\
\hline Tumour stage (ref. is stage $0-2$ ) & 0.371 & 0.647 & $0.690(0.141-3.371)$ \\
\hline
\end{tabular}

used to compare the mean PSQI scores between the two groups. Relationship of psychiatric morbidity, age, gender, marital status, presence of a NG tube, presence of tracheotomy tube, cancer treatment (chemotherapy, radiotherapy and/or surgical management), tumour stage, education, use of Naswar, family income and cigarette smoking was also analysed with the sleep quality by using the Chi-square test. Level of significance was considered as $<0.05$ and binary logistic regression was done to ascertain the significance of relationship between the study variables.

\section{RESULTS}

A total of 170 patients from E.N.T Department with head and neck tumours during the period of our study were taken and selected on the basis of inclusion and exclusion criteria. Out of 170 patients screened through PSQI, 58 $(34.1 \%)$ had good sleep quality, while 112 (65.9\%) had poor sleep quality. One hundred and eight patients $(63.5 \%)$ had significant psychiatric morbidity when assessed with $\mathrm{GHQ}$, while 62 (36.5\%) had scores less than that to qualify for presence of psychiatric morbidity. Mean age of the patients was $52.1 \pm 6.2$ years. Sixty $(35.3 \%)$ patients were females, while $110(64.7 \%)$ were males.

Mean PSQI score in patients without psychiatric morbidity was $6.2 \pm 3.2$; while in the patients with psychiatric morbidity, it was $9.4 \pm 4.6$ ( $p<0.001)$. Older age, family income and psychiatric morbidity were significantly related to poor sleep quality (Table I). Presence of psychiatric morbidity and surgical procedure performed were strongly related to poor quality of sleep when binary logistic regression was applied (Table II).

\section{DISCUSSION}

Sleep problems and mental health issues had been linked to cancers in various studies done in the recent past around the globe.4,7,9 Based upon PSQI score, $65.9 \%$ of the cancer patients included in this study suffered from poor quality of sleep. The present findings were in line with other similar studies done on the patients of head and neck tumours in other parts of the world.8,9 Patients with head and neck cancers pass through various issues that can impair their sleep, like pain, depression, obstructive sleep apnea, xerostomia and use of nicotine or alcohol.9 Reason behind these may be related to physical effects of tumour, radiation therapy, long course of illness and high expenditure.

Globally, it has been observed that mental health issues have been highly prevalent among the cancer patients. 15,16 Patients in this study showed similar pattern, and psychiatric morbidity was high among them, with $63.5 \%$ participants having GHQ score more than 3 . Individuals screened positive can be administered specific questionnaires or shown to psychiatrist for confirmation of diagnosis. Quality of sleep and psychiatric problems have a strong correlation. ${ }^{17}$ Sleeprelated problems and psychological issues aggravate each other and patient is entangled in this cycle till both the issues are addressed.18 Sleep is a complex biological function with psychological, physiological, neurological and social dimensions; so it needs discussion involving all these aspects. All these dimensions become altered to some extent when a person is suffering from some systemic illness. It is easier for the health professionals to recognise medical causes linked to sleep disturbances in the patients suffering from malignancies of head and neck region; but difficult for them to screen for psychological problems related with sleep issues.

Physical causes of these tumours may add to poor sleep among these individuals. Xerostomia and pressure effects can be troublesome for the patient and hinder in good quality of sleep.11,13 Head and neck cancer itself, its treatment, cosmetic disfigurement and slow treatment response may prone the patient towards psychological problems.12 Mental health problems add to the poor quality of life and can also be linked to the issues of noncompliance which can be counter-therapeutic. Thus, both sleep problems and psychological issues need to be recognised and treated in time for overall wellbeing of the individuals suffering from head and neck cancer.8,11 
Increasing age had been related with poor sleep quality among the patients of tumours of head and neck region in the past. ${ }^{11}$ Results in our study are similar to those studies. Surgical resection of tumour was a significant factor in causing poor sleep in our study. Though surgery is the main stay of treatment in these head and neck tumours; but still the trauma, pain and disfigurement associated with the surgery may cause direct effect on sleep quality and may indirectly contribute by increasing the psychiatric morbidity. Smoking and status of education were not related to poor sleep in the target population of this study. Recent literature also supports these findings. ${ }^{7}$

Poor quality of sleep had strong relation with low income. A study in 2010 produced similar results. ${ }^{18}$ Patients who have less affordability may be in more stress and apprehension due to huge burden of the disease treatment with limited income resources. ${ }^{18}$ Restriction and unable to perform the job duties and unemployment may contribute to these factors.

This study has few limitations as well. The major limitation is the use of psychometric tools for sleep quality and psychological wellbeing; which have been designed for screening instead of diagnosis. They were also not administered prior to the diagnosis of malignancy, and study design was not prospective so cause and effect relationship of variables could not be clear. Methodological issues also arise due to small sample size and use of self-administered psychometric tools. Hospital anxiety and depression scale (HADS) would have been more appropriate for this study, but we were screening for psychiatric morbidity not depression alone. It was not a population-based analysis, so lacks generalisability. Instead of a randomised sample of all the patients of head and neck tumours at various hospitals of Pakistan, patients from one public sector hospital were targeted. Chances of under- or over-reporting of symptoms also increase with the use of self-administered psychometrics like GHQ-12 and PSQI. Further studies with a larger sample size and use of locally developed more sophisticated psychometrics may prove helpful with more generalisability.

\section{CONCLUSION}

Patients suffering from head and neck tumours showed high presence of both poor sleep quality and psychiatric morbidity. Patients with presence of psychiatric morbidity or who had undergone surgical resection of tumour should be routinely screened for the sleep problems.

\section{REFERENCES}

1. National Sleep Foundation: Insomnia. Arlington, Va: National Sleep Foundation, 2014. Available online. Last accessed January 28, 2016.

2. Zubair UB, Malik Z, Ali U. Assessment of quality of sleep in pregnancy and socio-demographic factors associated with poor sleep quality in pregnancy. J Sleep Disor Treat Care 2016; 5:2.

3. Zubair UB, Butt B. Association of quality of sleep with cognitive decline among the patients of chronic kidney disease undergoing haemodialysis. J Ayub Med Coll Abbottabad 2017; 29:619-22.

4. Akman T, Yavuzsen T, Sevgen Z, Ellidokuz H, Yilmaz AU. Evaluation of sleep disorders in cancer patients based on Pittsburgh Sleep Quality Index. Eur J Cancer Care (Engl) 2015; 2:553-9.

5. Leblanc MF, Desjardins S, Desgagné A. Sleep problems in anxious and depressive older adults. Psychol Res Behav Manag 2015; 8:161-9.

6. Matos LL, Miranda GA, Cernea CR. Prevalence of oral and oropharyngeal human papilloma virus infection in Brazilian pop-ulation studies: A systematic review. Braz J Otorhinolaryngol 2015; 81:554-67.

7. Rigoni L, Bruhn RF, De Cicco R, Kanda JL, Matos LL. Quality of life impairment in patients with head and neck cancer and their caregivers: A comparative study. Braz J Otorhinolaryngol 2016; 82:680-6.

8. Lokker ME, Offerman MP, van der Velden LA, de Boer MF, Pruyn JF, Teunissen SC. Symptoms of patients with incurable head and neck cancer: Prevalence and impact on daily functioning. Head Neck 2013; 35:868-76.

9. Gandhi AK, Roy S, Thakar A, Sharma A, Mohanti BK. Symptom burden and quality of life in advanced head and neck cancer patients: AlIMS study of 100 patients. Indian J Palliat Care 2014; 20:189-93.

10. Faiz SA, Balachandran D, Hessel AC, Lei X, Beadle BM, William WN Jr, et al. Sleep-related breathing disorders in patients with tumours in the head and neck region. Oncologist 2014; 19:1200-6.

11. Ehrsson YT, Sundberg K, Laurell G, Eklöf AL. Head and neck cancer patients' perceptions of quality of life and how it is affected by the disease and enteral tube feeding during treatment. Ups J Med Sci 2015; 120:280-9.

12. Shuman AG, Duffy SA, Ronis DL, Garetz SL, McLean SA, Fowler KE, et al. Predictors of poor sleep quality among head and neck cancer patients. Laryngoscope 2010; 120:1166-72.

13. Safa M, Boroujerdi FG, Ardakani BMZ, Zadeh FH, Ardakini KMZ. Determining the relationship between sleep quality with mental health in 850 pulmonary patients. Eur Resp J 2016; 48:2338.

14. Hasselberg MJ, Porsteinsson AP, Boyle L, Parker KP. Subjective and objective measures of sleep quality in advanced cancer: A possible clinical marker for depression. J Sleep Disord Ther 2013; 2:135.

15. Jacobsen PB. Assessment of fatigue in cancer patients. J Natl Cancer Inst Monogr 2004; 32:93-7.

16. Ryan JL, Carroll JK, Ryan EP, Mustian KM, Fiscella K, Morrow GR. Mechanisms of cancer-related fatigue. Oncologist 2007; 12: 22-34.

17. Groenvold M, Petersen MA, Idler E, Bjorner JB, Fayers PM, Mouridsen HT. Psychological distress and fatigue predicted recurrence and survival in primary breast cancer patients. Breast Cancer Res Treat 2007; 105:209-19.

18. Naud K, Ouellet A, Brown C, Pasquier JC, Moutquin JM. Is sleep disturbed in pregnancy? J Obstet Gynaecol Can 2010; 32:28-34. 